\title{
Die Substitutionstherapie Opioidabhängiger in Deutschland: Auswirkungen der 3. BtMVVÄndV aus der Sicht substituierender Ärztinnen und Ärzte
}

\author{
Opioid Substitution Treatment in Germany: Physicians' View on \\ the Effects of the $3^{\text {rd }}$ Revision of the Narcotic Drugs Prescription \\ Ordinance
}

(c) (1) $\ominus$

\author{
Autoren \\ Kirsten Lehmann ${ }^{1 D}$, Silke Kuhn¹, Bernd Schulte', Hans-Günter Meyer-Thompson², Uwe Verthein ${ }^{1}$
}

\section{Institute}

1 Universitätsklinikum Hamburg-Eppendorf, Zentrum für Interdisziplinäre Suchtforschung der Universität Hamburg (ZIS), Hamburg, Deutschland

2 Substitutionsambulanz Altona, Asklepios Klinik Nord - Ochsenzoll, Klinik für Abhängigkeitserkrankungen, Hamburg, Deutschland

\section{Schlüsselwörter}

BtMVV, BtMVVÄndV, Evaluation, Opioidabhängigkeit,

Opioidsubstitutionstherapie, OST

\section{Key words}

NDPO, evaluation, opioid dependence, opioid substitution therapy, OST

online publiziert $\quad 01.03 .2021$

\section{Bibliografie}

Gesundheitswesen 2021; 83: 651-661

DOI 10.1055/a-1378-9249

ISSN 0941-3790

(C) 2021. The Author(s).

This is an open access article published by Thieme under the terms of the Creative Commons Attribution-NonDerivative-NonCommercial-License, permitting copying and reproduction so long as the original work is given appropriate credit. Contents may not be used for commercial purposes, or adapted, remixed, transformed or built upon. (https://creativecommons. org/licenses/by-nc-nd/4.0/)

Georg Thieme Verlag KG, Rüdigerstraße 14,

70469 Stuttgart, Germany

\section{Korrespondenzadresse}

Kirsten Lehmann

Universitätsklinikum Hamburg-Eppendorf

Zentrum für Interdisziplinäre Suchtforschung

der Universität Hamburg (ZIS)

Klinik für Psychiatrie und Psychotherapie

Martinistraße 52

20246 Hamburg

Deutschland

k.lehmann@uke.de

\section{ZUSAMMENFASSUNG}

Ziel der Studie Die weltweit effektivste Behandlungsform der Opioidabhängigkeit ist die Substitutionsbehandlung mit Opioiden (Opioidsubstitutionstherapie - OST). Diese Therapieform ist auch in Deutschland etabliert. Jedoch bestehen Versorgungslücken, v. a. in ländlichen Gebieten und einzelnen Bundesländern, was auf eine abnehmende Anzahl substituierender Ärztlnnen zurückzuführen ist. Mit der 3. Verordnung zur Änderung der Betäubungsmittel-Verschreibungsverordnung (3. BtMVVÄndV) in 2017 wurden ärztlich-therapeutische Aufgaben der OST in die Richtlinienkompetenz der Bundesärztekammer überführt. Die umfassende Reform der Rahmenbedingungen der OST führt zu einer stärkeren Rechtssicherheit dieser Behandlungsform. Die vorliegende Studie zielt darauf ab, die Auswirkungen der 3. BtMVVÄndV aus der Behandelndenperspektive zu analysieren. Methode Ein Fragebogen zu individuellen Erfahrungen mit den Veränderungen durch die 3. BtMVVÄndV wurde zwischen August und Dezember 2019 an 2503 substituierende ÄrztInnen in Deutschland sowie an 563 nicht oder länger nicht mehr substituierende ÄrztInnen in Hamburg, Bayern, Nordrhein-Westfalen sowie Sachsen verschickt. Die Versendung erfolgte über die Bundesopiumstelle sowie die Kassenärztlichen Vereinigungen der ausgewählten Bundesländer. Die Auswertung differenziert zwischen substituierenden Ärztlnnen mit und ohne suchtmedizinische Weiterbildung sowie zwischen städtischen und ländlichen Kreisen.

Ergebnisse Die Rücklaufquote substituierender ÄrztInnen lag bei $34,1 \%$. Das Durchschnittsalter betrug 57,9 $( \pm 8,7)$ Jahre und $64,5 \%$ waren männlich. Aus substitutionsärztlicher Sicht waren die relevantesten Änderungen der 3. BtMVVÄndV keine zeitliche Vorgabe für das Erreichen einer Opioidabstinenz (85,3\%), die neue Bewertung und Behandlung des Konsums weiterer psychotroper Substanzen (71,0\%), die Möglichkeit, die TakeHome-Verordnung auf 30 Tage auszudehnen (70,0\%) sowie die größere Rechtssicherheit (66,2\%). Die Ausweitung der Konsiliarbehandlung auf 10 PatientInnen erfuhr mit 14,8\% wenig Zustimmung. 36,7\% sah die Versorgung substituierter PatientInnen weder aktuell noch zukünftig gesichert.

Schlussfolgerung Die Neuerungen durch die 3. BtMVVÄndV wurden positiv aufgenommen und hinsichtlich der erhöhten 
Rechtssicherheit und therapeutischen Freiheiten als relevant eingestuft. Informationsbedarf besteht in ländlichen Gebieten, bei substituierenden Ärztlnnen ohne suchtmedizinische Weiterbildung sowie bei ÄrztInnen, die in der Vergangenheit substituierten, es aktuell jedoch nicht mehr tun.

\section{ABSTRACT}

Study aim Opioid substitution treatment (OST) is the most effective drug treatment for opioid dependence worldwide. This form of therapy is also well established in Germany. Nevertheless, there are gaps in the provision of care, especially in rural areas and some states, due to a decreasing number of physicians involved in implementing the substitution programs. The $3^{\text {rd }}$ revision of the Narcotic Drugs Prescription Ordinance (NDPO), which came into force in 2017, transferred medical therapeutic tasks of OST to the policy-making power of the German Medical Association. This comprehensive reform of the general conditions for OST led to greater legal certainty for this form of treatment. The present study aimed to analyze the effects of the $3^{\text {rd }}$ revision of the Narcotic Drugs Prescription Ordinance from the providers' perspective.

Methods Between August and December 2019, a questionnaire on individual experiences with the changes implemented in $3^{\text {rd }}$ revision of the Narcotic Drugs Prescription Ordinance was sent by the Federal Opium Agency and the Associations of Statutory Health Insurance Physicians of the chosen federal states to 2,503 physicians implementing the substitution program in Germany as well as 563 physicians in Hamburg, Bavaria, North Rhine-Westphalia and Saxony who were not or no longer involved in this field of medical practice.The evaluation distinguished between physicians with and without further training in addiction medicine and between urban and rural districts.

Results The response rate of physicians was $34.1 \%$. The average age was $57.9( \pm 8.7)$ years, and $64.5 \%$ were male. The most relevant changes of the NDPO revision were found to be no time limit for achieving opioid abstinence (85.3\%), new assessment and treatment using additional psychotropic substances $(71.0 \%)$, extending take-home regulation to a maximum of 30 days (70.0\%) and greater legal certainty (66.2\%). Widening of consultative care up to 10 patients met with little approval (14.8\%); $36.7 \%$ did not believe that care of substituted patients was assured either now or in the future.

Conclusions The NDPO revisions were considered to be relevant in terms of increased legal certainty and treatment liberties. Information was needed in rural areas, among physicians who carried out substitution therapy without advanced training in addiction medicine and physicians no longer involved in substitution therapy.

\section{Einleitung}

Die Behandlung opioidabhängiger Menschen mit Substitutionsmedikamenten ist weltweit die verbreitetste und effektivste Therapieoption der Opioidabhängigkeit [1]. Die Opioidsubstitutionstherapie (opioid substitution treatment - OST) zielt darauf ab, Drogenbeschaffung sowie den illegalen Opioidkonsum zu unterbinden. Sie reduziert die Mortalität, den Gebrauch von illegalen Substanzen sowie das HIV- und Hepatitis-C-Risikoverhalten und wirkt sich positiv auf die soziale Stabilisierung und (Re-)Integration der PatientInnen aus [2-7]. Weiterhin entsteht durch eine OST eine enge ärztliche Anbindung und somit ein regelmäßiger Zugang zur medizinischen Grundversorgung.

Die gesetzliche Grundlage für die Etablierung der Opioidsubstitution in Deutschland als eine anerkannte und von den Krankenkassen finanzierte Behandlungsform wurde 1992 durch die Verankerung im Betäubungsmittelgesetz (BtMG) gelegt. Während anfangs lediglich Levomethadon als Substitutionsmedikament zur Verfügung stand, ermöglichen heute diverse Wirkstoffe wie Levomethadon, Buprenorphin, retardiertes Morphin, Codein/Dihydrocodein oder Diamorphin mit ihren spezifischen Wirkungs- und Nebenwirkungsprofilen eine stärker individualisierte Substitutionsbehandlung von Opioidabhängigen [8-9].

Da Anfang der 1990er-Jahre noch keine Leitlinien für die Substitutionsbehandlung Opioidabhängiger vorlagen, gaben die Betäubungsmittel-Verschreibungsverordnung (BtMVV) sowie die Richtlinien über neue Untersuchungs-und Behandlungsmethoden (NUB-Richtlinien) die Therapieinhalte und -ziele vor. Die Bundesärztekammer (BÄK) veröffentlichte erstmals 2002 eine Richtlinie zur Substitutionsbehand- lung, die in 2009/2010 überarbeitet und in 2017 letztmalig novelliert wurde [10-11].

In Deutschland befindet sich etwa die Hälfte aller opioidabhängigen Personen in einer Substitutionsbehandlung. In 2019 wurden 78.700 substituierte Opioidabhängige von 2607 substituierenden ÄrztInnen behandelt [8, 12]. Die Anzahl der Substituierten wie auch die der Behandelnden war in den letzten 10 Jahren relativ konstant bzw. auf die substituierenden ÄrztInnen bezogen leicht abnehmend. Dem Bericht zum Substitutionsregister des BfArM [8] ist zu entnehmen, dass v. a. in ländlichen Gebieten schon derzeit keine substituierenden ÄrztInnen tätig sind [8]. Perspektivisch werden in naher Zukunft eine Vielzahl der Substituierenden altersbedingt ihre Praxen aufgeben und grundsätzlich Probleme haben, Nachfolgerlnnen zu finden $[13,14]$. Zudem führten in einigen Bundesländern Verstöße gegen das Abstinenzziel oder die Duldung des Konsums weiterer psychoaktiver Substanzen (,Beikonsum') zu strafrechtlichen Verfahren gegen substituierende ÄrztInnen, was die Gewinnung neuer ÄrztInnen für diese Art der Behandlung von Opioidabhängigen zusätzlich erschwert hat [15].

Den Fortbestand und die Qualität der Substitutionsbehandlung Opioidabhängiger in Deutschland auch in Zukunft durch eine ausreichende Anzahl substituierender ÄrztInnen zu sichern, war ein Hauptmotiv für den Beschluss der 3. Verordnung zur Änderung der Betäubungsmittel-Verschreibungsverordnung (3. BtMVVÄndV) im Mai 2017. Übergeordnete Ziele dieser Novellierung waren die Verbesserung der Rechtssicherheit für substituierende ÄrztInnen und die Durchführung der Substitutionsbehandlung entsprechend dem neuesten wissenschaftlichen Stand und praktischen Erfordernis- 
sen. Erstmals werden die medizinisch-therapeutischen Aspekte der Substitutionsbehandlung ausschließlich durch die Richtlinie der BÄK geregelt [16], während sich die BtMVV weitgehend auf die Verordnung der zugelassenen Substitutionsmedikamente und Aspekte der Betäubungsmittel (BtM)-Sicherheit beschränkt [17]. In die Richtlinienkompetenz der BÄK wurden im Wesentlichen die Therapieziele, die Voraussetzungen für die Einleitung und Fortführung einer Substitutionsbehandlung sowie die Erstellung eines Therapiekonzepts überführt $[16,18]$. Mit der Veröffentlichung dieser Richtlinie im Bundesanzeiger trat die 3. BtMVVÄndV am 02.10.2017 in Kraft.

Eine wichtige Änderung ist die Bewertung ärztlich-therapeutischer Sachverhalte durch die Landesärztekammern (LÄK), was die bisherige Strafbewehrung von Verstößen gegen die Therapieziele aufhebt. Die LÄK sind nun, wie bei anderen ärztlichen Tätigkeiten auch, Ansprechpartner bei eventuellen Verstößen gegen die ärztlichen Berufspflichten im Rahmen der Substitutionsbehandlung. Die Opioidabstinenz der PatientInnen als ein Behandlungsziel (neben weiteren), soll ohne zeitliche Vorgaben angestrebt werden. Für die Aufgabe von zeitlichen Vorgaben zur Erreichung einer Opioidabstinenz im Rahmen der Substitutionsbehandlung liegt ausreichend Evidenz vor $[3,19,20]$. In der deutschen PREMOS-Studie konnten lediglich $8 \%$ der Teilnehmenden eine temporäre und $4 \%$ eine stabile Opioidabstinenz innerhalb von 6 Jahren erreichen [6].

Bezüglich der Bewertung und Behandlung des Konsums weiterer psychotroper Substanzen einschließlich Alkohol (,Beikonsum`) gibt die 3. BtMVVÄndV den BehandlerInnen die therapeutische Freiheit, die Substitutionsbehandlung auch bei fortgesetztem Konsum anderer psychotroper Substanzen weiterzuführen, wobei deren Reduktion als ein Therapieziel verfolgt werden sollte. Es gilt aber nicht mehr, dass keine Substanzen gebraucht werden dürfen, deren Konsum nach Art und Menge den Zweck der Substitution gefährden. Ein Therapieabbruch oder eine Beendigung der Substitutionsbehandlung ohne alternative Anschlussbehandlung sollte möglichst vermieden werden, unter anderem, da ein Abbruch der Substitutionsbehandlung das Mortalitätsrisiko im Fall einer Wiederaufnahme des Drogenkonsums aufgrund geringerer Opioidtoleranzen erhöht [20-22].

Eine psychosoziale Betreuung (PSB) soll den substituierten Opioidabhängigen weiterhin regelhaft empfohlen werden, ist aber nicht mehr grundsätzlich erforderlich, um eine Substitutionsbehandlung durchführen zu dürfen. Neuere Literaturübersichten kamen zu dem Ergebnis, dass eine PSB keinen signifikanten Einfluss auf den Erfolg einer Substitutionsbehandlung hat [7, 23].

Die Möglichkeit, die Take-Home-Verordnung (von einer Apotheke ausgehändigte Substitutionsmittel zur eigenverantwortlichen Einnahme zu Hause) von 7 auf bis zu 30 Tage sowie die Ausnahme vom Sichtbezug (unmittelbare Substitutionsmitteleinnahme unter Aufsicht) von 2 auf 5 Tage auszudehnen, soll den substituierten PatientInnen mehr Teilhabe am gesellschaftlichen Leben ermöglichen und somit bspw. den (Wieder-)Einstieg in das Berufsleben erleichtern. Eine weitere Änderung besteht darin, dass ÄrztInnen ohne eine suchtmedizinische Weiterbildung anstatt wie bisher 3 nunmehr 10 PatientInnen unter Betreuung durch einen Konsiliarius substituieren können. Während noch von Opiatabhängigen gesprochen wurde, wurde der Terminus 2017 in Opioidabhängige geändert. Dies eröffnet die Möglichkeit, alle Opioidabhän- gigen zu substituieren, also auch Menschen, die von ärztlich verschriebenen (halb-)synthetischen Opioiden wie bspw. Fentanyl, Tramadol oder Tilidin abhängig geworden sind [24]. Die Voraussetzung für eine Substitutionsbehandlung ist die missbräuchliche Verwendung der Opioide. Eine Opioidabhängigkeit als Nebenwirkung einer regulären Behandlung mit Opioiden, z. B. im Rahmen von Schmerzbehandlungen, sollte dagegen in erster Linie durch Reduktion oder schrittweisen Entzug der Opioide behandelt werden.

Im Juni 2018 hat die Gesundheitsministerkonferenz der Länder einstimmig die Evaluation der Neuregelung zur substitutionsgestützten Behandlung opioidabhängiger Menschen beschlossen [25]. Das vom Zentrum für Interdisziplinäre Suchtforschung der Universität Hamburg (ZIS) ausgearbeitete Evaluationskonzept umfasst modular aufgebaute quantitative und qualitative Erhebungsstrategien sowie Sekundärdatenanalysen über einen Zeitraum von 3 Jahren. Hierbei werden mögliche Effekte aus Sicht von Patientlnnen sowie VersorgerInnen (ÄrztInnen, ApothekerInnen) untersucht. In diesem Artikel werden die Ergebnisse der ersten bundesweit durchgeführten Befragung von substituierenden ÄrztInnen vorgestellt. Im Mittelpunkt stehen dabei die Darstellung der Charakteristika der substituierenden ÄrztInnen unter besonderer Berücksichtigung des Alters und Art der Durchführung der Substitution (mit oder ohne suchtmedizinische Weiterbildung), die Bewertung und praktische Umsetzung der neuen Richtlinien zur Substitution sowie die Analyse versorgungsrelevanter Unterschiede zwischen städtischen und ländlichen Gebieten.

\section{Methodik}

\section{Studiendesign}

Die Befragung fand als Teilprojekt im Rahmen der Evaluation der 3. Verordnung zur Änderung der BtMVV vom 22.05.2017 („Substitutions-Novelle“) - EVASUNO im Zeitraum von August bis Dezember 2019 statt. Von der Bundesopiumstelle des BfArM wurden deutschlandweit alle substituierenden ÄrztInnen (Stichtag 12.08.2018) angeschrieben und um die Beantwortung eines Fragebogens gebeten. Darüber hinaus erfolgte über den Zugangsweg der regionalen Kassenärztlichen Vereinigungen (KVen) eine Befragung von nicht oder schon länger nicht mehr substituierenden ÄrztInnen in den durch das Evaluationsprojekt definierten Modellregionen Hamburg, Bayern, Nordrhein-Westfalen (Westfalen-Lippe, Nordrhein) sowie Sachsen. Die hierfür angeschriebenen ÄrztInnen hatten in der Vergangenheit eine Substitutionsgenehmigung erhalten. Es handelte sich bei beiden Vorgehensweisen um anonyme Befragungen, die aggregiert ausgewertet wurden. Der zuständigen Ethikkommission der Landesärztekammer Hamburg wurde das Projektvorhaben zur Begutachtung vorgelegt. Das Vorgehen wurde als nicht ethikrelevant eingestuft.

Die Bundesopiumstelle sowie die KVen der vier Modellregionen erhielten vorfrankierte Briefumschläge mit einem jeweils angepassten Anschreiben, dem Fragebogen und einem Rückumschlag. Die zuvor frankierten und verschlossenen Umschläge wurden von der Bundesopiumstelle und den KVen mit Adressaufklebern versehen und verschickt. Alle ÄrztInnen hatten darüber hinaus die Möglich- 
keit, den jeweiligen Fragebogen auch webbasiert, über die OnlineUmfrage-Applikation LimeSurvey, zu beantworten.

Parallel zu dieser Befragung wurden alle KVen in Deutschland gebeten, einen Kurzfragebogen u. a. zum Alter der substituierenden ÄrztInnen zu beantworten.

\section{Erhebungsinstrument}

Der Fragebogen wurde in Kooperation mit substituierenden ÄrztInnen und dem für Substitutionsfragen zuständigen Bereichsleiter der BÄK entwickelt. Daraufhin erfolgte eine Pilotisierung mit dem Studienteam bekannten substituierenden ÄrztInnen, die zu einer leichten Überarbeitung des Fragebogens führte. Die Inhalte der Befragung bezogen sich auf strukturelle Merkmale ( z. B. ärztliches Fachgebiet, Qualifikation zur Substitution, Anzahl Substituierter, Art der Praxis) sowie auf die in der 3. BtMVVÄndV beschlossenen Neuerungen. Die Änderungen der 3. BtMVVÄndV sollten hinsichtlich der Relevanz für die eigene Substitutionsbehandlung kategorial beurteilt werden. Hierfür wurden die Änderungen vorgegeben:

- Größere Rechtssicherheit durch Bewertung ärztlich-therapeutischer Sachverhalte durch die Landesärztekammern.

- Substitution von PatientInnen mit (ausschließlicher) Abhängigkeit von synthetischen Opioiden möglich.

- Keine zeitlichen Vorgaben für das Erreichen einer Opioidabstinenz.

- Neue Bewertung des Umgangs mit dem Konsum weiterer psychotroper Substanzen (,Beikonsum').

- Konsiliarbehandlung von 10 PatientInnen möglich.

- Ausnahme vom Sichtbezug auf 5 Tage ausgedehnt.

- Take-Home-Verordnung auf 30 Tage ausgedehnt.

- Herausnahme der Erforderlichkeit psychosozialer Betreuungsmaßnahmen.

\section{Stichprobe}

Über die Bundesopiumstelle wurden 2.503 Ärztlnnen (Rücklaufquote: 34,1\%) und über die KVen 563 ÄrztInnen (Rücklaufquote: $22,2 \%$ angeschrieben. Lediglich 11 StudienteilnehmerInnen nutzten die webbasierte Antwortmöglichkeit. Für die Überprüfung der Repräsentativität der erhaltenen Fragebögen wurde die Teilnahmequote der substituierenden ÄrztInnen pro Bundesland der Quote der durch die Bundesopiumstelle versendeten Fragebögen pro Bundesland gegenübergestellt. Insgesamt 16 der bundesweit 17 Landes-KVen nahmen an der Befragung teil. 12 KVen übermittelten in anonymer Form aktuelle Altersangaben ihrer insgesamt 1629 substituierenden ÄrztInnen. Diese Angaben wurden ebenfalls für die Abschätzung der Repräsentativität der Befragung verwendet.

\section{Auswertung}

Die Auswertungen erfolgten quantitativ mithilfe des Statistikprogramms IBM ${ }^{\circledR}$ SPSS $^{\circledR}$ Statistics 22 (IBM Corporation 2013). Die regionale Zuordnung der Praxiseinrichtungen nach städtischen und ländlichen Bereichen, den Kreisregionen der Bundesrepublik Deutschland, erfolgte anhand der ersten 3 Ziffern der Postleitzahl der jeweiligen Einrichtungen [26]. Kreisregionen werden vom Bundesinstitut für Bau-, Stadt- und Raumforschung (BBSR) in siedlungsstrukturelle Kreistypen unterteilt. Auf diese Weise können 4 sied- lungsstrukturelle Kreistypen unterschieden werden: kreisfreie Großstädte, städtische Kreise, ländliche Kreise mit Verdichtungsansätzen sowie dünn besiedelte ländliche Kreise. Diese Einordnung berücksichtigt, dass die Lebensbedingungen der Kreise sowie ihre Veränderungen von der Entwicklung sowie Struktur der jeweiligen Region, vielmehr des Regionstyps abhängig sind [27]. In einigen Fällen war es aufgrund identischer Postleitzahlziffern der ersten 3 Stellen nicht möglich, eine eindeutige Zuordnung zu einem Kreistypen vorzunehmen. Für weitergehende Analysen wurden die beiden Kreistypen ,ländlicher Kreis mit Verdichtungsansätzen‘ und ,dünn besiedelter ländlicher Kreis‘ zu dem Kreis ,ländlicher Kreis‘ zusammengefasst.

\section{Ergebnisse}

\section{Regionale Repräsentativität}

Der Auflistung der Bundesopiumstelle war zu entnehmen, wie viel Prozent der insgesamt verschickten 2503 Fragebögen an substituierende ÄrztInnen in den unterschiedlichen Bundesländern verschickt wurden. So wurden z. B. 28,2\% der 2.503 Fragebögen an Behandlerlnnen in Nordrhein-Westfalen versandt, verglichen mit $1,7 \%$ an BehandlerInnen in Sachsen. Dementsprechend war es möglich, die Teilnahmequoten an der Befragung pro Bundesland mit den Quoten der tatsächlich versandten Fragebögen zu vergleichen. Es zeigte sich eine nahezu identische Verteilung der verschickten Fragebögen und der Teilnahmequoten pro Bundesland. Kein Bundesland ist deutlich über- oder unterrepräsentiert, so dass Auswertungen für die einzelnen Bundesländer erfolgen konnten.

ÄrztInnen mit einer suchtmedizinischen Qualifikation sind in dieser Umfrage mit 86,3\% überrepräsentiert im Vergleich zu 78,2\% laut dem Bericht zum Substitutionsregister für das Jahr 2019 [8]. ÄrztInnen die 3 oder weniger PatientInnen substituieren, sind in der vorliegenden Erhebung im Vergleich zum selben Bericht zum Substitutionsregister unterrepräsentiert (15 vs. $27 \%$ ) und diejenigen, die mehr als 50 PatientInnen substituieren überrepräsentiert (31 vs. $23 \%$ ). Die Repräsentativität der über die KVen angeschriebenen Ärztlnnen kann nicht überprüft werden. Die Repräsentativität der Befragung kann, wenn auch eingeschränkt, zusätzlich an den Altersangaben der KVen überprüft werden. 12 KV-Bezirke übermittelten in anonymer Form aktuelle Altersangaben ihrer insgesamt 1629 substituierenden Ärztlnnen. Die Ärztlnnen waren im Mittel 58,6 (SD = 8,9) Jahre alt. In unserer Stichprobe lag der Altersmittelwert der ÄrztInnen aus den entsprechenden $12 \mathrm{KV}$-Bezirken bei $58,2(S D=8,7)$ Jahren.

\section{Aktuell substituierende Ärztinnen und Ärzte}

Von den 833 aktuell substituierenden Ärztlnnen waren knapp zwei Drittel männlich. Das Durchschnittsalter der Befragten betrug 57,9 Jahre (Spannweite: 30-80), 19,1\% waren über 65 Jahre alt. Mehrheitlich wurde als ärztliches Fachgebiet Allgemeinmedizin und/ oder Innere Medizin angegeben. Die allermeisten arbeiteten als niedergelassene ÄrztInnen, entweder in einer eigenen Praxis und dort überwiegend alleine oder in einer Gemeinschaftspraxis, Praxisgemeinschaft oder einem medizinischen Versorgungszentrum (MVZ) mit substituierenden KollegInnen ( $>$ Tab. 1). Von denen in anderen Einrichtungsformen Tätigen arbeiteten 44 in Justizvoll- 
- Tab. 1 Charakteristika der substituierenden ÄrztInnen mit und ohne suchtmedizinische Weiterbildung

\begin{tabular}{|c|c|c|c|c|}
\hline & \multicolumn{2}{|c|}{ Substituierende ÄrztInnen } & \multirow[b]{2}{*}{$\begin{array}{l}\text { Gesamt } \\
(\mathrm{N}=\mathbf{8 2 7})\end{array}$} & \multirow[b]{2}{*}{$\begin{array}{l}\text { Statistik } \\
\text { Chi' }{ }^{2} \text {-Test } \\
\text { \#T-Test }\end{array}$} \\
\hline & $\begin{array}{l}\text { mit suchtmed. } \\
\text { Weiterbildung } \\
\text { ( } N=714)\end{array}$ & $\begin{array}{l}\text { ohne suchtmed. } \\
\text { Weiterbildung } \\
\text { ( } \mathrm{N}=113)\end{array}$ & & \\
\hline Alter in Jahren $(M, S D)(N=809)$ & $58,0(8,8)$ & $57,2(8,1)$ & $57,9(8,7)$ & $p=0,368 \#$ \\
\hline \multicolumn{5}{|l|}{ Geschlecht $(N=808)$} \\
\hline männlich & $444(63,6 \%)$ & $77(70,0 \%)$ & $521(64,5 \%)$ & \\
\hline weiblich & $254(36,4 \%)$ & $33(30,0 \%)$ & $287(35,5 \%)$ & $\mathrm{p}<0,193$ \\
\hline \multicolumn{5}{|l|}{ Siedlungsstrukturelle Kreistypen $(N=627)$} \\
\hline Kreisfreie Großstädte & $225(41,1 \%)$ & $9(11,3 \%)$ & $234(37,3 \%)$ & \\
\hline Städtische Kreise & $185(33,8 \%)$ & $34(42,5 \%)$ & $219(34,9 \%)$ & \\
\hline Ländliche Kreise & $137(25,0 \%)$ & $37(46,3 \%)$ & $174(27,8 \%)$ & $\mathrm{p}<0,001$ \\
\hline \multicolumn{5}{|l|}{ Fachgebiet $(\mathrm{N}=822)$} \\
\hline Allgemeinmedizin & $434(61,2 \%)$ & $80(70,8 \%)$ & $514(62,5 \%)$ & \\
\hline Innere Medizin & $85(12,0 \%)$ & $22(19,5 \%)$ & $107(13,0 \%)$ & \\
\hline Psychiatrie/Neurologie & $140(19,7 \%)$ & $6(5,3 \%)^{*}$ & $146(17,8 \%)$ & \\
\hline Anderes Fachgebiet & $50(7,1 \%)$ & $5(4,4 \%)$ & $55(6,7 \%)$ & $\mathrm{p}<0,001$ \\
\hline \multicolumn{5}{|l|}{ Arbeitsstruktur $(\mathrm{N}=808)$} \\
\hline Einzelpraxis & $259(37,2 \%)$ & $64(57,7 \%)$ & $323(40,0 \%)$ & \\
\hline Gemeinschaftspraxis, Praxisgemeinschaft, MVZ & $258(37,0 \%)$ & $37(33,3 \%)$ & $295(36,5 \%)$ & \\
\hline Substitutionsambulanz & $77(11,0 \%)$ & 0 & $77(9,5 \%)$ & \\
\hline Psychiatrische Institutsambulanz & $39(5,6 \%)$ & $1(0,9 \%)$ & $40(5,0 \%)$ & \\
\hline Andere Versorgungseinrichtung ( z. B. JVA, Gesundheitsamt) & $64(9,2 \%)$ & $9(8,1 \%)$ & $73(9,0 \%)$ & $\mathrm{p}<0,001$ \\
\hline Jahre in Substitution tätig $(\mathrm{M}, \mathrm{SD})(\mathrm{N}=818)$ & $15,3(8,9)$ & $8,6(7,2)$ & $14,4(9,0)$ & $\mathrm{p}<0,001 \#$ \\
\hline Alter bei Beginn der Substitution (M, SD) $(N=802)$ & $42,8(7,3)$ & $48,8(8,0)$ & $43,6(7,7)$ & $\mathrm{p}<0,001 \#$ \\
\hline PatientInnenanzahl (M, SD) (1. Quartal 2019) $(\mathrm{N}=814)$ & $53,2(54,2)$ & $3,5(3,4)$ & $46,4(53,1)$ & $\mathrm{p}<0,001 \#$ \\
\hline \multicolumn{5}{|l|}{ Substitutionsmittel (Mehrfachangaben) } \\
\hline Levomethadon & $648(91,3 \%)$ & $67(59,8 \%)$ & $715(87,0 \%)$ & $\mathrm{p}<0,001$ \\
\hline Buprenorphin & $626(88,0 \%)$ & $61(54,5 \%)$ & $687(83,5 \%)$ & $\mathrm{p}<0,001$ \\
\hline Razemisches Methadon & $544(76,5 \%)$ & $39(34,8 \%)$ & $583(70,8 \%)$ & $\mathrm{p}<0,001$ \\
\hline Retardiertes Morphin & $260(36,6 \%)$ & $6(5,4 \%)$ & $266(32,3 \%)$ & $\mathrm{p}<0,001$ \\
\hline Buprenorphin-Depot & $78(11,0 \%)$ & $3(2,7 \%)$ & $81(9,8 \%)$ & $p=0,006$ \\
\hline Codein/Dihydrocodein & $40(5,6 \%)$ & $2(1,8 \%)$ & $42(5,1 \%)$ & $p=0,086$ \\
\hline \multicolumn{5}{|l|}{$\begin{array}{l}\text { Durchschnittliche Anzahl unterschiedlicher Substitutionsmittel } \\
(M, S D)(N=827)\end{array}$} \\
\hline Änderung der Anzahl Substituierter im Vergleich zum Vorjahr (N=822) & $3,1(1,1)$ & $1,6(0,7)$ & $2,9(1,2)$ & $\mathrm{p}<0,001 \#$ \\
\hline Verringert & $77(10,8 \%)$ & $6(5,4 \%)$ & $83(10,1 \%)$ & $p=0,196$ \\
\hline In etwa gleich geblieben & $472(66,4 \%)$ & $80(72,1 \%)$ & $552(67,2 \%)$ & \\
\hline Erhöht & $162(22,8 \%)$ & $25(22,5 \%)$ & $187(22,7 \%)$ & \\
\hline
\end{tabular}

zugsanstalten. Drei Viertel der Befragten waren in städtischen Gebieten tätig. Die durchschnittliche Anzahl substituierter PatientInnen lag bei 46,4 (Spannweite: 1-550). Die meisten Befragten gaben an, dass die Anzahl von ihnen Substituierter im Vergleich zum Vorjahr stabil geblieben war. Die am häufigsten eingesetzten Substitutionsmedikamente waren Levomethadon, Buprenorphin, razemisches Methadon und retardiertes Morphin ( $\triangleright$ Tab. 1).
Von den befragten ÄrztInnen verfügten 714 (86,3\%) über eine suchtmedizinische Zusatzqualifikation (Weiterbildung in suchtmedizinischer Grundversorgung [ $\mathrm{N}=650$ ] oder Fachkunde Sucht [N =64]). Der Vergleich von ÄrztInnen mit und ohne suchtmedizinische Zusatzqualifikation zeigte einige bedeutsame Unterschiede ( Tab. 1). Ärztlnnen ohne suchtmedizinische Weiterbildung substituierten (bei vergleichbarem Durchschnittsalter) seit deutlich kürzerer Zeit, waren somit bei Beginn ihrer Substitutionstätigkeit 


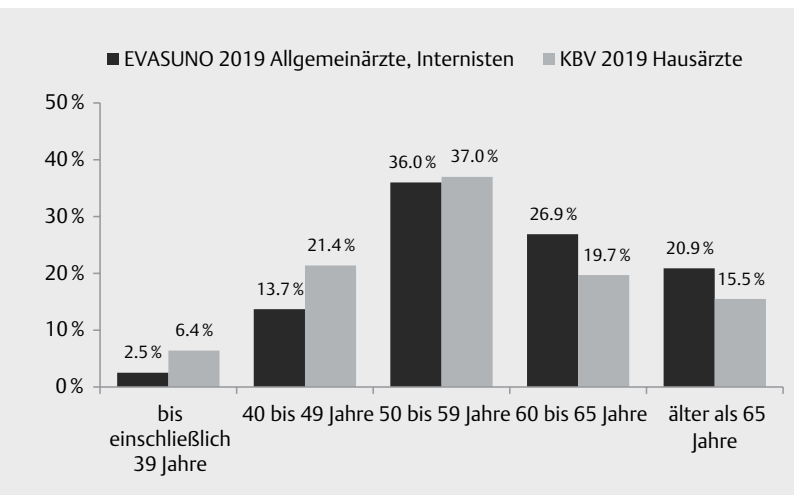

- Abb. 1 Vergleich der Altersangaben der befragten substituierenden AllgemeinärztInnen/InternistInnen in eigenen Praxen, Praxisgemeinschaften oder Gemeinschaftspraxen mit denen aller niedergelassenen HausärztInnen nach Angaben der Kassenärztlichen Bundesvereinigung (KBV), Alterskategorien entsprechend der KBV-Statistik.

älter, häufiger FachärztInnen für Allgemeinmedizin und/oder Innere Medizin und praktizierten seltener in kreisfreien Großstädten als jene mit suchtmedizinischer Weiterbildung. Von den 113 ÄrztInnen, die unter der Konsiliarregelung tätig waren, nutzten bisher lediglich 6 (5,4\%) die Möglichkeit, ihre PatientInnenzahl auf maximal 10 zu erhöhen, während 73 (65,2\%) ÄrztInnen weiterhin 1 bis $3 \mathrm{~Pa}$ tientInnen substituierten; die restlichen $29,4 \%$ substituierten zwischen 4 und 9 Patientlnnen.

22 der 855 Ärztlnnen mit einer Substitutionsgenehmigung gaben an, aktuell nicht zu substituieren. Diese waren im Durchschnitt 58,3 Jahre alt (Spannweite: 37 bis 74 ) und 23,8\% waren weiblich. In der Mehrzahl (63,6\%) verfügten sie über keine suchtmedizinische Zusatzqualifikation und substituierten vormals im Rahmen der Konsiliarregelung. Eine fachärztliche Ausbildung für Psychiatrie und Psychotherapie/Neurologie besaßen 31,8\%.

\section{Alter}

Der Vergleich mit den Daten der Kassenärztlichen Bundesvereinigung (KBV) ${ }^{1}$ zeigte, dass die substituierenden AllgemeinärztInnen/ Internistlnnen in der Altersklasse ab 60 Jahre überrepräsentiert und in der Altersklasse von 49 Jahre und jünger unterrepräsentiert waren [28] (॰ Abb. 1).

\section{Beurteilung der Änderungen der Betäubungsmittel- Verschreibungsverordnung von städtischen und ländlichen Regionen im Vergleich}

Signifikante Unterschiede zwischen Ärztlnnen in städtischen und ländlichen Regionen bezogen sich auf die durchschnittliche Anzahl der von ihnen substituierten PatientInnen im 1. Quartal 2019 (kreisfreie Großstadt: 63,9; städtischer Kreis: 40,3; ländlicher Kreis: $36,7 ; F=16,58, p<0,001)$ sowie auf den suchtmedizinischen Weiterbildungsstatus (kreisfreie Großstadt: 96,2\%; städtischer Kreis:

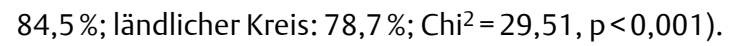

1 Die KBV unterteilt die niedergelassenen ÄrztInnen in Hausärztlnnen, FrauenärztInnen, AugenärztInnen usw., ohne explizite Nennung von AllgemeinärztInnen oder Internistlnnen.
Die $>$ Tab. 2 zeigt die Bewertung der Änderungen der BtMVV sowie damit zusammenhängende relevante Ergebnisse bezogen auf die PSB und Versorgungsstrukturen. Mehr als drei Viertel der befragten substituierenden Ärztlnnen fühlten sich über die Änderungen der BtMVV ausreichend informiert, deutlich mehr in den kreisfreien Großstädten. Während sich ÄrztInnen mit einer suchtmedizinischen Weiterbildung zu 81,3\% ausreichend informiert fühlten, waren es ohne suchtmedizinische Weiterbildung lediglich 54,9\% (Chi² $=39,3, p<0,001)$. Keine zeitliche Vorgabe für das Erreichen einer Opioidabstinenz, die neue Bewertung des Konsums weiterer psychotroper Substanzen einschließlich Alkohol (,Beikonsum'), die Möglichkeit, die Take-Home-Verordnung auf 30 Tage auszudehnen sowie die größere Rechtssicherheit wurden am relevantesten eingeschätzt. Etwas über ein Drittel der Befragten sah die Versorgung substituierter PatientInnen weder aktuell noch zukünftig gesichert. In den ländlichen und städtischen Kreisen war die Einschätzung negativer als in den kreisfreien Großstädten.

Die Mehrheit der subsituierenden Einrichtungen/Praxen befand sich nicht weiter als $5 \mathrm{~km}$ voneinander entfernt, was vornehmlich auf großstädtische Regionen zutrifft. Lediglich 9,4\% der Befragten berichteten Entfernungen von mehr als $30 \mathrm{~km}$. In den ländlichen Kreisen waren die Entfernungen zwischen den substituierenden Einrichtungen signifikant größer. Die ÄrztInnen wurden gefragt, wie viele ihrer PatientInnen eine PSB benötigen und wie viele diese tatsächlich in Anspruch nehmen. Ein ausgeglichenes Verhältnis gaben 41,7\% der Befragten an, während 33,7 \% einen höheren Bedarf an PSB-Plätzen hatten. Demgegenüber berichteten 24,6\%, dass sie eigentlich weniger PSB-Plätze benötigen, als zum Zeitpunkt der Befragung vorhanden waren. Dieses Verhältnis zeigte sich mehr oder weniger in allen Kreistypen. Bezüglich der vereinfachten Möglichkeit, bspw. auch in Pflegeeinrichtungen substituieren zu können, zeigten sich hingegen Unterschiede zwischen den Kreistypen. Die Überlegungen, die Substitutionsbehandlung durch die Entstehung weiterer Substitutionsambulanzen oder Abteilungen in psychiatrischen Kliniken sowie durch eine verstärkte Einbindung von FachärztInnen für Psychiatrie und Psychotherapie/Neurologie zu sichern, wurde je nach Kreistyp ebenfalls uneinheitlich bewertet.

Keine Unterschiede zwischen den Kreistypen fanden sich hinsichtlich des Alters der substituierenden ÄrztInnen, ihres Geschlechts sowie ihrer fachärztlichen Qualifikation. Ebenso zeigten sich keine Unterschiede in Bezug auf die Änderung der Anzahl Substituierter im Vergleich zum Vorjahr sowie der Anzahl der Jahre, welche die ÄrztInnen bereits Substitutionsbehandlungen durchführten.

\section{Mögliche Beendigung der Substitutionstätigkeit und Bedingungen für die eigenständige Durchführung der Substitutionsbehandlung}

42,8\% der befragten substituierenden ÄrztInnen gaben an, noch nie eine Beendigung ihrer Substitutionstätigkeit in Betracht gezogen zu haben. Als Hauptmotive für eine mögliche Aufgabe der Substitutionsbehandlung wurden Altersgründe, ein (zu) hoher bürokratischer Aufwand und Beweggründe, welche die eigene Praxisorganisation betreffen genannt ( $\triangleright$ Tab. 3 ). Lediglich $7 \%$ gaben finanzielle Gründe an. Zwischen den ÄrztInnen mit und ohne suchtmedizinische Weiterbildung fanden sich keine signifikanten Unterschiede. Für die meisten ÄrztInnen, die unter der Konsiliarregelung 
- Tab. 2 Bewertung der Änderungen der BtMVV durch befragte substituierende ÄrztInnen und relevante Parameter der Versorgung substituierter PatientInnen nach Kreistypen.

\begin{tabular}{|c|c|c|c|c|c|}
\hline & $\begin{array}{l}\text { Kreisfreie } \\
\text { Großstadt N = } 234\end{array}$ & $\begin{array}{l}\text { Städtischer } \\
\text { Kreis } \mathbf{N}=\mathbf{2 2 0}\end{array}$ & $\begin{array}{l}\text { Ländlicher } \\
\text { Kreis N=174 }\end{array}$ & $\begin{array}{l}\text { Gesamt } \\
N=628\end{array}$ & $\begin{array}{l}\text { Statistik } \\
\text { Chi²-Test }^{2}\end{array}$ \\
\hline \multicolumn{6}{|l|}{$\begin{array}{l}\text { Ausreichende Information über Änderungen } \\
\text { der BtMVV vorhanden }(N=620)\end{array}$} \\
\hline Ja & $198(85,3 \%)$ & $162(75,3 \%)$ & $128(74,0 \%)$ & $488(78,7 \%)$ & $p=0,007$ \\
\hline Nein & $34(14,7 \%)$ & $53(24,7 \%)$ & $45(26,0 \%)$ & $132(21,3 \%)$ & \\
\hline \multicolumn{6}{|l|}{ Relevante Änderungen (Mehrfachangaben) } \\
\hline $\begin{array}{l}\text { Keine zeitliche Vorgabe für das Erreichen einer } \\
\text { Opioidabstinenz }\end{array}$ & $198(86,8 \%)$ & $182(84,3 \%)$ & $143(84,6 \%)$ & $523(85,3 \%)$ & $p=0,711$ \\
\hline Neue Bewertung Beikonsum* & $175(76,8 \%)$ & $144(66,7 \%)$ & $116(68,6 \%)$ & $435(71,0 \%)$ & $p=0,048$ \\
\hline Take-Home-Verordnung: max. 30 Tage & $155(68,0 \%)$ & $158(73,1 \%)$ & $116(68,6 \%)$ & $429(70,0 \%)$ & $p=0,447$ \\
\hline Größere Rechtssicherheit & $163(71,8 \%)$ & $136(63,0 \%)$ & $106(62,7 \%)$ & $405(66,2 \%)$ & $p=0,078$ \\
\hline Herausnahme der Erforderlichkeit von PSB & $122(53,5 \%)$ & $88(40,7 \%)$ & $68(40,2 \%)$ & $278(45,4 \%)$ & $p=0,008$ \\
\hline Ausnahme vom Sichtbezug: 5 Tage & $63(27,6 \%)$ & $76(35,2 \%)$ & $61(36,1 \%)$ & $200(32,6 \%)$ & $p=0,125$ \\
\hline $\begin{array}{l}\text { Substitution von PatientInnen mit primärer } \\
\text { Abhängigkeit von synthetischen Opioiden }\end{array}$ & $74(32,5 \%)$ & $48(22,2 \%)$ & $54(32,0 \%)$ & $176(28,7 \%)$ & $p=0,032$ \\
\hline Konsiliarbehandlung max.10 PatientInnen & $21(9,2 \%)$ & $31(14,4 \%)$ & $39(23,1 \%)$ & $91(14,8 \%)$ & $p=0,001$ \\
\hline \multicolumn{6}{|l|}{$\begin{array}{l}\text { Änderung der eigenen Arbeit durch die Reform } \\
(\mathrm{N}=623)\end{array}$} \\
\hline Eher positiv & $124(53,0 \%)$ & $104(48,4 \%)$ & $69(39,7 \%)$ & $297(47,7 \%)$ & \\
\hline Eher negativ & $6(2,6 \%)$ & $5(2,3 \%)$ & $3(1,7 \%)$ & $14(2,2 \%)$ & $p=0,207$ \\
\hline Nicht verändert & $71(30,3 \%)$ & $70(32,6 \%)$ & $71(40,8 \%)$ & $212(34,0 \%)$ & \\
\hline Noch nicht beurteilbar & $33(14,1 \%)$ & $36(16,7 \%)$ & $31(17,8 \%)$ & $100(16,1 \%)$ & \\
\hline \multicolumn{6}{|l|}{$\begin{array}{l}\text { Versorgung substituierter PatientInnen im } \\
\text { Umfeld gesichert }(N=610)\end{array}$} \\
\hline Aktuell und in der Zukunft gesichert & $77(33,8 \%)$ & $50(23,1 \%)$ & $29(17,5 \%)$ & $156(25,6 \%)$ & \\
\hline $\begin{array}{l}\text { Aktuell gesichert, aber innerhalb der nächsten } 5 \\
\text { Jahre gefährdet }\end{array}$ & $100(43,9 \%)$ & $86(39,8 \%)$ & $44(26,5 \%)$ & $230(37,7 \%)$ & \\
\hline Weder aktuell noch zukünftig gesichert & $51(22,4 \%)$ & $80(37,0 \%)$ & $93(56,0 \%)$ & $224(36,7 \%)$ & $p<0,001$ \\
\hline \multicolumn{6}{|l|}{$\begin{array}{l}\text { Entfernung zur nächsten substituierenden } \\
\text { Einrichtung }(\mathrm{N}=625)\end{array}$} \\
\hline In der Nähe, im Umkreis von ca. 5 km & $198(84,6 \%)$ & $108(49,8 \%)$ & $48(27,6 \%)$ & $354(56,6 \%)$ & $\mathrm{p}<0,001$ \\
\hline Ca. über 5 bis $15 \mathrm{~km}$ entfernt & $31(13,2 \%)$ & $59(27,2 \%)$ & $30(17,2 \%)$ & $120(19,2 \%)$ & \\
\hline Ca. über 15 bis $30 \mathrm{~km}$ entfernt & $2(0,9 \%)$ & $44(20,3 \%)$ & $46(26,4 \%)$ & $92(14,7 \%)$ & \\
\hline Über 30 km entfernt & $3(1,3 \%)$ & $6(2,8 \%)$ & $50(28,7 \%)$ & $59(9,4 \%)$ & \\
\hline
\end{tabular}

tätig waren, ist die Substitutionsbehandlung ein randständiger Bereich ihrer ärztlichen Tätigkeit. 35,9\% konnten sich vorstellen, zukünftig eine suchtmedizinische Zusatzweiterbildung zu absolvieren und eigenständig zu substituieren.

\section{Ärztinnen und Ärzte, die nicht oder nicht mehr substituieren}

Von den 125 nicht substituierenden ÄrztInnen aus den 4 Modellregionen, die über eine Zulassung zur Substitution verfügten, waren 72,0\% männlich und der Altersdurchschnitt lag bei 57,9 Jahren $(S D=9,5)$. Lediglich $56,8 \%$ verfügten über eine suchtmedizinische Zusatzqualifikation. 81,5\% hatten in der Vergangenheit schon einmal Substitutionsbehandlungen durchgeführt. Über die Hälfte dieser ÄrztInnen gab die Substitutionstätigkeit aufgrund eines zu hohen zeitlichen sowie Dokumentationsaufwands auf. Gut ein Drittel meinte, Substituierte würden nicht mit den anderen Patientln- nen der Praxis harmonieren ( $\vee$ Tab. 4). Für jeweils knapp die Hälfte der ÄrztInnen wären Voraussetzungen für die Aufnahme einer Substitutionstätigkeit, dass diese angemessener honoriert und noch mehr von bestehenden Regularien befreit werden müsste.

\section{Diskussion und Schlussfolgerungen}

Das Ziel dieser Untersuchung war es, substituierende und nicht (mehr) substituierende ÄrztInnen zu ihrer Bewertung der Veränderungen der 3. BtMVVÄndV, die zum Befragungszeitpunkt seit etwa 2 Jahren in Kraft war, sowie deren Relevanz für die praktische Arbeit zu befragen.

Die Informationen zu den Änderungen der BtMVV wurden über ÄK und KVen, Verbände und Gesellschaften, über Materialien der Substitutionsmittelhersteller aber auch unter den Ärztlnnen gut verbreitet. Trotz regionaler Unterschiede fühlt sich die große Mehr- 
> Tab. 3 Von substituierenden ÄrztInnen genannte Gründe für eine mögliche Beendigung der Substitutionstätigkeit und Bedingungen für eine eigenständige Durchführung der Substitutionsbehandlung von ÄrztInnen, die unter Konsiliarregelung tätig waren.

\begin{tabular}{|l|c|}
\hline Substituierende Ärztlnnen (gesamt) & N (\%) \\
\hline Gründe, aus denen eine Beendigung der Substitutionstätigkeit überlegt wurde (Mehrfachangaben) & $218(26,8 \%)$ \\
\hline Fortgeschrittenes Alter & $194(23,9 \%)$ \\
\hline Zu hoher Aufwand/zu bürokratisch & $127(15,7 \%)$ \\
\hline Praxisorganisation & $57(7,0 \%)$ \\
\hline Finanzielle Gründe & $85(10,5 \%)$ \\
\hline Andere, z. B. Rechtsunsicherheit, juristische Ermittlungen, Einbrüche, Diebstähle, aggressive Patientlnnen & \\
\hline Ärztlnnen, die unter Konsiliarregelung tätig waren (Mehrfachangaben) & \\
\hline Gründe, warum eine Substitutionstätigkeit nur unter Konsiliarregelung durchgeführt wurde & $82(77,4 \%)$ \\
\hline Substitution soll kein Schwerpunkt werden & $52(50,0 \%)$ \\
\hline Substitution nur, weil Patientlnnen sonst nicht versorgt wären & $24(23,1 \%)$ \\
\hline Nicht genügend Kapazitäten, um eigenständig zu substituieren & $4(3,8 \%)$ \\
\hline Tätig vorwiegend als Urlaubsvertretung oder aus kollegialen Gründen & \\
\hline Bedingungen, um zukünftig selbstständig zu substituieren (Mehrfachangaben) & $49(53,8 \%)$ \\
\hline Eigene Praxisorganisation müsste verändert werden & $44(48,4 \%)$ \\
\hline Eigene Fortbildung müsste erfolgen & $35(38,0 \%)$ \\
\hline Substitutionsbehandlung müsste stärker von Regularien befreit werden & $26(28,6 \%)$ \\
\hline Netzwerk von ärztlichen Kolleglnnen & $26(28,3 \%)$ \\
\hline Substitutionsbehandlung müsste angemessenerer honoriert werden & \\
\hline
\end{tabular}

> Tab. 4 Gründe für die Beendigung und Voraussetzungen für die (Wieder-)Aufnahme der Substitutionstätigkeit bei nicht substituierenden ÄrztInnen.

\begin{tabular}{|c|c|}
\hline ÄrztInnen, die nicht oder nicht mehr substituierten $(N=124)$ & $\mathrm{N}(\%)$ \\
\hline \multicolumn{2}{|l|}{ Gründe für Aufgabe der Substitution (N=101) (Mehrfachangaben) } \\
\hline Substitutionsbehandlung ist zu aufwendig (Zeit, Dokumentation) & $50(53,8 \%)$ \\
\hline SubstitutionspatientInnen harmonieren nicht mit anderen PatientInnen & $33(35,5 \%)$ \\
\hline Substitutionsbehandlung ist rechtlich zu unsicher & $29(31,2 \%)$ \\
\hline Substitutionsbehandlung wird nicht ausreichend honoriert & $28(30,1 \%)$ \\
\hline Organisatorisch nicht mehr möglich & $27(29,0 \%)$ \\
\hline Nicht genügend eigene Kapazitäten & $18(19,4 \%)$ \\
\hline Altersgründe/Aufgabe der Praxis & $6(6,5 \%)$ \\
\hline Anderes & $41(44,1 \%)$ \\
\hline \multicolumn{2}{|l|}{ Wenn noch niemals substituiert, ist die Aufnahme der Substitution vorstellbar? ( $N=23$ ) (Mehrfachangaben) } \\
\hline Grundsätzlich vorstellbar & $4(17,4 \%)$ \\
\hline Nur PatientInnen mit Opioidanalgetika-Abhängigkeit (nicht Heroin) & $3(13,0 \%)$ \\
\hline Wenn PatientInnen nicht anderweitig versorgt werden können & $2(8,7 \%)$ \\
\hline Hat sich bisher noch nicht ergeben & $2(8,7 \%)$ \\
\hline Nur vorübergehend bzw. in Vertretung & $3(13,0 \%)$ \\
\hline \multicolumn{2}{|l|}{ Was müsste sich verändern, damit Substitutionstätigkeit aufgenommen würde? ( $\mathrm{N}=104)$ (Mehrfachangaben) } \\
\hline Angemesseneres Honorar & $51(49,0 \%)$ \\
\hline Weniger Regularien & $51(49,0 \%)$ \\
\hline Änderung der eigenen Praxisorganisation (und ggf. Struktur) & $50(48,1 \%)$ \\
\hline Netzwerkbildung aus weiteren ärztlichen KollegInnen & $32(30,8 \%)$ \\
\hline Kontinuierliche Konsultationsmöglichkeit einer/s erfahrenen Suchtmedizinerln oder eine Fachambulanz & $31(29,8 \%)$ \\
\hline Mehr eigene Fortbildung & $22(21,2 \%)$ \\
\hline Interessierte Kollegln in der Praxis & $11(10,6 \%)$ \\
\hline
\end{tabular}


heit der befragten StudienteilnehmerInnen ausreichend gut über die Änderung der BtMVV informiert. Dass sich substituierende ÄrztInnen in kreisfreien Großstädten insgesamt besser informiert fühlten, lässt sich mit den höheren Anteilen an ÄrztInnen mit suchtmedizinischer Weiterbildung und einer ggf. besseren Vernetzung aufgrund einer höheren Praxisdichte in kreisfreien Großstädten erklären.

Die Aufgabe einer zeitlichen Vorgabe für das Erreichen einer Opioidabstinenz war für die substituierenden ÄrztInnen mit Abstand die bedeutendste Änderung der BtMVV, gefolgt von den Neuerungen im Umgang mit Beikonsum, der Ausweitung der TakeHome-Verordnungen und der grundsätzlich größeren Rechtssicherheit. Diese Neuerungen scheinen für die substituierende Ärzteschaft von besonderer Bedeutung, da Verstöße v. a. bei der Fortführung der Substitution bei nachgewiesenem Konsum weiterer psychotroper Substanzen einschließlich Alkohol (,Beikonsum') in der Vergangenheit zu Gerichtsverfahren und Verurteilungen zu Geldstrafen bis hin zum Entzug der Approbation geführt hatten [29]. Die Substitutionsbehandlung hat sich zu einer langjährigen und oft lebenslangen Therapie entwickelt, die nur ein geringer Prozentsatz an Substituierten regulär mit einer stabilen Abstinenz von Opioiden beendet [6]. Die Anforderungen, sich möglicherweise für eine therapeutisch angemessene langjährige Substitutionsbehandlung gegenüber KVen, Medizinischen Diensten der Krankenversicherung oder Krankenkassen rechtfertigen zu müssen und ggf. stabil substituierte Opioidabhängige in eine abstinenzorientierte Behandlung zu drängen, ist entfallen. Es gilt für die Behandelnden nicht mehr, wie Backmund noch 2015 ausführte: „Der Arzt ist somit täglich damit konfrontiert, zwischen möglicher Strafverfolgung und Behandlung nach heutigem Stand der Wissenschaft zum Wohle der Patienten zu entscheiden“ [30, S.15].

Die Ausdehnung der Take-Home-Vergabe auf 30 Tage ermöglicht es den substituierenden ÄrztInnen, flexibel auf die Lebenssituation der stabil Substituierten zu reagieren. Berufliche Reisetätigkeiten oder Urlaubswünsche können nunmehr unter Beibehaltung der Substitution ohne organisatorischen Mehraufwand realisiert werden. Damit wird ein wichtiges Therapieziel der Substitutionsbehandlung, die soziale (Re-)Integration, also eine verbesserte Teilhabe der PatientInnen am Leben in der Gesellschaft und am Arbeitsleben, nicht mehr durch Verordnungen behindert.

Gemessen an den Ergebnissen dieser Studie, scheint die durchschnittliche Verfügbarkeit von PSB-Plätzen in Deutschland ausreichend zu sein. Sie unterliegt zwar regionalen Schwankungen, aber folgt nicht den häufig angenommenen Stadt-Land-Unterschieden bei der Versorgung von Opioidabhängigen. Da sich die Bedarfe an PSB-Plätzen in allen Kreistypen in einem vergleichbaren Verhältnis widerspiegelten, können ländliche oder kleinstädtische Gebiete, bezogen auf PSB-Plätze, genauso über- wie unterversorgt sein. Allerdings kann keine Aussage darüber getroffen werden, inwiefern sich PSB-Angebote in Wohnortnähe der substituierten PatientInnen befinden.

Es fällt auf, dass die Herausnahme der Erforderlichkeit der PSB von weniger als der Hälfte der Befragten als relevant eingestuft wird. Hier spiegelt sich insgesamt eine ambivalente Haltung der Ärzteschaft zum Stellenwert der PSB wider. Allerdings muss angemerkt werden, dass die PSB ein Sammelbegriff unterschiedlicher psychosozialer Maßnahmen ist. Dementsprechend empfehlen die
Leitlinien der Weltgesundheitsorganisation (World Health Organization - WHO), substituierten PatientInnen den Zugang zur PSB zu ermöglichen, die Substitutionsbehandlung jedoch nicht von der Teilnahme an einer PSB abhängig zu machen [1]. Die Möglichkeit, abhängige PatientInnen, die primär (halb-)synthetische Opioide (z. B. opioidhaltige Schmerzmittel) missbräuchlich konsumieren, in eine reguläre Opioidsubstitution überführen zu können, ist den Studienteilnehmerlnnen bekannt, jedoch werden die Auswirkungen auf die Routineversorgung als gering eingeschätzt. Die gefürchtete ,Opioidwelle', wie sie aus den USA bekannt ist, wird in dieser Form in Deutschland nicht erwartet.

Substituierende ÄrztInnen ohne suchtmedizinische Weiterbildung betreuen nur $1 \%$ aller substituierten PatientInnen [8]. Da lediglich 5,4\% dieser ÄrztInnen in der vorliegenden Studie ihre PatientInnenzahl auf bis zu 10 erhöht haben, scheint die Möglichkeit der Aufstockung von einer untergeordneten Bedeutung zu sein. Es ist anzunehmen, dass sie zumeist lediglich aus kollegialer Gefälligkeit bekannten ÄrztInnen oder PatientInnen gegenüber substituieren. Gleichwohl könnte sich gut ein Drittel der Ärztlnnen vorstellen, sich suchtmedizinisch weiterzubilden. Neben hemmenden Bedingungen, die grundsätzlich auch von suchmedizinisch weitergebildeten ÄrztInnen geäußert wurden, wie zu viele Regularien oder eine nicht ausreichende Vergütung, wurde auch zu wenig Zeit für eine Weiterbildung oder der Wunsch nach einem Netzwerk von substituierenden KollegInnen genannt. Dies adressiert zum einen an die Landesärztekammern in ihren Funktionen als Fortbildungsträger sowie an bestehende Netzwerke substituierender ÄrztInnen oder Qualitätszirkel, ihre Tätigkeit zu bewerben.

Die Möglichkeit der Abgabe von Substitutionsmedikamenten in Alten- und Pflegeheimen oder Hospizen, über ambulante Pflegedienste oder in Rehabilitationseinrichtungen und Gesundheitsämtern wird zurückhaltend beurteilt, obgleich diese in Zukunft einer größeren Rolle bei der flächendeckenden Absicherung der Substitutionsbehandlung zukommen. Der Bedarf an Behandlungsmöglichkeiten in Alten- und Pflegeheimen, Hospizen oder über ambulante Pflegedienste wird zukünftig ansteigen, da der Altersdurchschnitt der PatientInnen und damit entsprechende Versorgungsbedarfe zunehmen. Hierbei sind möglicherweise bisher nicht ausreichend geklärte haftungs- und vertragsrechtliche Fragen relevant. Rechtsverbindliche Vertragsmuster, wie die Mustervereinbarung zum Sichtbezug von Substitutionsmitteln in der Apotheke, die von der Bundesvereinigung Deutscher Apothekerverbände e. V. (ABDA) auf ihrer Internetseite zur Verfügung gestellt wird, liegen bisher für die Zusammenarbeit von substituierenden ÄrztInnen und Pflegeeinrichtungen nicht vor.

Die Änderungen der BtMVV waren das Ergebnis eines jahrelangen aufwendigen Prozesses im Bundesgesundheitsministerium und in der Bundesärztekammer und entsprachen mit dem Bericht der Bundesärztekammer in 2017 dem aktuellen Stand der Erkenntnisse der medizinischen Wissenschaft [11]. Gleichwohl beurteilte nur knapp die Hälfte der substituierenden ÄrztInnen die Alltagsrelevanz der neuen Regularien positiv, während auch 2 Jahre nach Inkrafttreten der 3. BtMVVÄndV die andere Hälfte der BehandlerInnen keine alltagsrelevanten Änderungen angab bzw. diese (noch) nicht beurteilen konnte. Im ländlichen Raum ist die Bewertung der Alltagsrelevanz noch deutlich weniger positiv. Dieses Ergebnis könnte auf eine anhaltende Unsicherheit in der Umsetzung 
der Richtlinien hindeuten, auf eine geringe Motivation, die eigene Substitutionspraxis an die veränderten Möglichkeiten anzupassen oder auch darauf, dass weitere Faktoren hinsichtlich der Zufriedenheit mit der Substitutionsbehandlung eine Rolle spielen. Die Stigmatisierung von OpioidpatientInnen in der Allgemeinbevölkerung sowie der Substitutionsbehandlung könnte nach wie vor das mangelnde Interesse der Ärzteschaft an dieser Form der ärztlichen Tätigkeit erklären. Das Versäumnis, die Suchtbehandlung als integralen Bestandteil der ärztlichen Ausbildung zu verstehen und ,zu bewerben ' zeigt sich auch im Vergleich des Durchschnittsalters der substituierenden ÄrztInnen mit den bei der KBV registrierten HausärztInnen, wobei sich das Nachwuchsproblem unter den Substituierenden akzentuierter darstellt. Das Aufzeigen von Perspektiven sowie zielgerichtete Angebote für NachwuchsärztInnen, wie bspw. die Möglichkeit zur Teilnahme an Qualitätszirkeln oder eine verbesserte Vernetzung von Nachwuchsgruppen, könnten dazu beitragen, die zukünftige Bereitschaft für die Opioidsubstitution zu steigern. Die drohende Unterversorgung von Substituierten durch die Aufgabe substituierender Praxen aufgrund der Erreichung des Renteneintrittsalters der substituierenden ÄrztInnen muss auch im Kontext der Tatsache gesehen werden, dass niedergelassene Hausärztlnnen zunehmend und insbesondere in ländlichen Regionen Schwierigkeiten haben, NachfolgerInnen für ihre Kassensitze zu finden $[13,14]$. Daher sollte die Möglichkeit, auch andere Institutionen wie Gesundheitsämter oder Suchtberatungsstellen sowie PSB-Einrichtungen in die Substitutionsbehandlung einzubinden, weiter ausgebaut werden.

\section{Limitationen und Stärken}

An der bundesweiten Befragung beteiligten sich 34,1\% aller aktuell substituierenden ÄrztInnen. Die Rücklaufquote ist im Vergleich mit ähnlichen Studien, deren Rücklauf zwischen 14,7 und 24,4\% lag, als zufriedenstellend zu bewerten [31. In einer in 2013 veröffentlichten Studie von Schulte und KollegInnen zu den strukturellen Barrieren in der Substitutionsbehandlung, in der ebenfalls alle substituierenden ÄrztInnen in Deutschland angeschrieben wurden, betrug der Rücklauf 25,5 \% [34]. Der höhere Rücklauf in der vorliegenden Befragung deutet darauf hin, dass die Auswirkungen der 3. BtMVVÄndV für die substituierenden Ärztlnnen ein relevantes Thema darstellt. Ebenso wie in der Studie von Schulte und Kolleglnnen war der Rücklauf unter den ÄrztInnen, die mehr als 50 substituierte PatientInnen behandelten, höher im Vergleich zu Ärztlnnen mit bis zu 3 PatientInnen. Der Anteil derjenigen, der über eine suchttherapeutische Weiterbildung verfügte, ist unter den Teilnehmenden der Befragung höher als unter allen substituierenden ÄrztInnen in Deutschland. Dies lässt sich möglicherweise darauf zurückführen, dass die Auswirkungen der 3. BtMVVÄndV für Ärztınnen mit mehr PatientInnen eine höhere Relevanz haben als für diejenigen, die nach der Konsiliarregelung arbeiten. Somit ist denkbar, dass sich vermehrt ÄrztInnen, denen die 3. BtMVVÄndV bekannt ist und die ein besonderes Interesse an den Standards der Substitutionsbehandlung haben, an dieser Befragung beteiligten, sodass eine Stichprobenverzerrung der Studienpopulation aufgrund selektiver Effekte (Selektionsbias) möglich ist. Positiv hervorzuheben ist die regionale Repräsentativität der Stichprobe. Die Teilnahmequote pro Bundesland entspricht dem Anteil aller über die Bundesopiumstelle verschickten Fragebögen. Ebenso ist der tatsächliche Altersmittelwert der substituierenden ÄrztInnen aus 12 KVen (65\% aller substituierenden Ärztlnnen) mit dem Altersmittelwert der Studie für diese KVen nahezu identisch.

\section{Finanzielle Förderung}

Die vorliegende Arbeit ist Teil einer vom Bundesministerium für Gesundheit (BMG) finanziell geförderten Studie (Förderkennzeichen ZMVI1-2519DSM206).

\section{Interessenkonflikt}

KL erhielt eine Reisekostenerstattung sowie eine Kostenübernahme von Teilnahmegebühren und Übernachtungskosten von pcm scientific sowie die Übernahme von Konferenzteilnahmegebühren von Sanofi-Aventis. SK hat keine Interessenkonflikte. BS hat von CAMURUS Fördermittel sowie Reisekosten erhalten. H-GM-T ist Redakteur des Internetforums Substitutionspraxis. Er erhält dafür von den auf der Homepage ausgewiesenen Firmen keine Zuwendungen. UV erhielt innerhalb der letzten 3 Jahre Vortrags-, Beratungshonorar und Reisekostenerstattungen von Mundipharma GmbH sowie finanzielle Projektförderung und Reisekostenerstattungen von CAMURUS GmbH.

Literatur

[1] World Health Organization (WHO). Guidelines for the Psychosocially Assisted Pharmacological Treatment of Opioid Dependence. 2009. https://www.who.int/substance_abuse/publications/opioid_dependence_guidelines.pdf?ua $=1$

[2] Degenhardt L, Bucello C, Mathers B et al. Mortality among regular or dependent users of heroin and other opioids: a systematic review and meta-analysis of cohort studies. Addiction 2010; 106: 32-51

[3] Mattick R, Breen C, Kimber J et al. Methadone maintenance therapy versus no opioid replacement therapy for opioid dependence. Cochrane Database of Systematic Reviews 2009; 3. doi:10.1002/14651858.CD002209.pub2

[4] Busch M, Haas S, Weigl M et al. Langzeitsubstitutionsbehandlung Opioidabhängiger. In: (DIMDI) DIfMDul ed. Schriftenreihe Health Technology Assessment (HTA) in der Bundesrepublik Deutschland. Köln: 2007

[5] Soyka M, Träder A, Klotsche J et al. Mortalität in der langfristigen Substitution: Häufigkeiten, Ursachen und Prädiktoren. Suchtmedizin in Forschung und Praxis 2011; 13: 247-252

[6] Wittchen H, Bühringer G, Rehm J et al. Der Verlauf und Ausgang von Substitutionspatienten unter den aktuellen Bedingungen der deutschen Substitutionsversorgung nach 6 Jahren. Suchtmedizin in Forschung und Praxis 2011; 13: 232-246

[7] Amato L, Minozzi S, Davoli M et al. Psychosocial combined with agonist maintenance treatments versus agonist maintenance treatments alone for treatment of opioid dependence Cochrane Database of Systematic Reviews 2011;10. doi:10.1002/14651858. CD004147.pub4

[8] Bundesinstitut für Arzneimittel und Medizinprodukte (BfArM). Bericht zum Substitutionsregister: Januar 2020. 2020. https://www.bfarm.de/ SharedDocs/Downloads/DE/Bundesopiumstelle/SubstitReg/Subst_Bericht2020.pdf

[9] Fischer G, Kayer B. Substanzabhängigkeit vom Morphintyp - State-ofthe-Art der Erhaltungstherapie mit synthetischen Opioiden. Psychiatrie \& Psychotherapie 2006; 2: 39-54 
[10] Kunstmann W, Specka M, Wodarz N et al. Substitutions-Richtlinie der Bundesärztekammer: Basis für eine bessere Versorgung. Deutsches Ärzteblatt 2019; 116: A-880. https://www.aerzteblatt.de/pdf. asp?id $=206993$

[11] Bundesärztekammer. Bericht der Bundesärztekammer zur Feststellung des aktuellen Standes der Erkenntnisse der medizinischen Wissenschaft in einer Richtlinie zur Durchführung der substitutionsgestützten Behandlung pioidabhängiger - Methodik und Ergebnisbewertung.In Berlin: Bundesärztekammer; 2017; https://www.bundesaerztekammer.de/fileadmin/user_upload/downloads/pdf-Ordner/Sucht/ Methodenbericht_Substitution.pdf

[12] Kraus L, Seitz N, Schulte B et al. Schätzung der Anzahl von Personen mit einer Opioidabhängigkeit. Deusches Ärzteblatt Int 2019; 116: 137-143. doi:10.3238/arztebl.2019.0137

[13] Sachverständigenrat zur Begutachtung der Entwicklung im Gesundheitswesens. Bedarfsgerechte Versorgung - Perspektiven für ländliche Regionen und ausgewählte Leistungsbereiche. In: 2014; https://www. svr-gesundheit.de/index.php?id $=465$

[14] Robert Koch-Institut Hrsg. Gesundheitsberichterstattung des Bundes gemeinsam getragen von RKI und Destatis. Gesundheit in Deutschland. In Berlin: RKI; 2015

[15] Ullmann R. Zur Strafverfolgung substituierender Ärzte. In, Bundesverband aeVDA-HJ, Hrsg. Alternativer Drogen- und Suchtbericht. 2014; https://alternativer-drogenbericht.de/zur-strafverfolgung-substituierender-aerzte/

[16] Bundesärztekammer. Richtlinie der Bundesärztekammer zur Durchführung der substitutionsgestützten Behandlung Opioidabhängiger In: Berlin: Bundesärztekammer; 2017; https://www.bundesaerztekammer.de/fileadmin/user_upload/downloads/pdf-Ordner/RL/ Substitution.pdf

[17] Bundesrat. Verordnung der Bundesregierung: Dritte Verordnung zur Änderung der Betäubungsmittel-Verschreibungsverordnung. 2017. Drucksache 222/217, 215.203.217, G; https://www.bundesrat.de/ SharedDocs/drucksachen/2017/0201-0300/2222-2017.pdf?_ blob $=$ publicationFile $\& \mathrm{v}=2019$

[18] Bühring P. Substitution opioidabhängiger Patienten: Aufwertung ärztlicher Kompetenz. Deutsches Ärzteblatt 2017; 114: A-1794; https://www.aerzteblatt.de/archiv/193765/Substitution-opioidabhaengiger-Patienten-Aufwertung-aerztlicher-Kompetenz

[19] Joseph H, Stancliff S, Langrod J. Methadone maintenance treatment (MMT): a review of historical and clinical issues. Mt Sinai J Med 2000; 67: 347-364

[20] Cornish R, Macleod J, Strang J et al. Risk of death during and after opiate substitution treatment in primary care: prospective observational study in UK. BMJ 2010; 341: c5475

[21] Degenhardt L, Larney S, Kimber J et al. The impact of opioid substitution therapy on mortality post-release from prison: retrospective data linkage study. Addiction 2014; 109: 1306-1317
[22] Sordo L, Barrio G, Bravo M et al. Mortality risk during and after opioid substitution treatment: systematic review and meta-analysis of cohort studies. British Medical Journal 2017; 357: j1550. doi:10.1136/bmj. j1550

[23] Dugosh K, Abraham A, Seymour B et al. A Systematic Review on the Use of Psychosocial Interventions in Conjunction With Medications for the Treatment of Opioid Addiction. Journal of Addiction Medicine 2016; 10: 93-103

[24] Nielsen S, Larance B, Degenhardt L et al. Opioid agonist treatment for pharmaceutical opioid dependent people. Cochrane Database of Systematic Reviews 2016; 5. doi:10.1002/14651858.CD011117.pub2

[25] Gesundheitsministerkonferenz (GMK). Beschlüsse der 91. GMK. TOP: 10.18 Evaluation der Neuregelung zur substitutionsgestützten Behandlung opioidabhängiger Menschen 2018. https://www. gmkonline. de $/$ Beschluesse. $h$ tml id $=727 \&$ jahr $=2018$

[26] Wiwald Data Services GmbH. Kostenlose Liste deutscher Postleitzahlen und zugehöriger Orte. In; o. J: https://www.wiwald.com/ds/ kostenlose-liste-deutscher-postleitzahlen-und-zugehoeriger-orte/id/ ww-german-postal-codes

[27] Bundesinstitut für Bau- S-uRB. INKAR - Indikatoren und Karten zur Raum- und Stadtentwicklung. In: Bonn; 2019; https://www.inkar.de/

[28] Kassenärztliche Bundesvereinigung (KBV). Gesundheitsdaten 2019: Ambulante Versorgung. 2019. https://gesundheitsdaten.kbv.de/cms/ html/16396.php

[29] Ullmann R. Strafrechtlicher Umgang mit substituierenden Ärzten aus suchtmedizinischer Sicht. Akzeptanzorientierte Drogenarbeit/ Acceptance-Oriented Drug Work. 2010; 7: 1-12

[30] Backmund M. Update Substitutionsbehandlung. HIV\&more 2015; 4 : 14-17

[31] Klingenberg A, Broge B, Herholz $\mathrm{H}$ et al. Hausarztzentrierte Versorgung aus Sicht der teilnehmenden Ärzte. Aktuelle Situation und Perspektiven. Medizinische Klinik 2010; 105: 89-95. doi:https://link. springer.com/article/10.1007/s00063-010-1012-8

[32] Götzmann L, Dutta M, Scheuer E et al. Psychosomatische Forschung in der Grundversorgung - Idealvorstellungen und Realität. Praxis 2002; 91: 1111-1115. doi:https://econtent.hogrefe.com/doi/ pdf/10.1024/0369-8394.91.25.1111

[33] Koch K, Gehrmann U, Sawicki P. Primärärztliche Versorgung in Deutschland im internationalen Vergleich. Ergebnisse einer strukturvalidierten Ärztebefragung. Deutsches Ärzteblatt. 2007; 104: 2584-2592

[34] Schulte B, Schmidt C, Kuhnigk O et al. Structural barriers in the context of opiate substitution treatment in Germany - a survey among physicians in primary care. Substance abuse treatment, prevention, and policy 2013; 8 